\title{
THE ONLY GENUS ZERO $n$-MANIFOLD IS $\mathbf{S}^{n}$
}

\author{
MASSIMO FERRI AND CARLO GAGLIARDI ${ }^{1}$
}

\begin{abstract}
All $n$-manifolds of regular genus zero, i.e. admitting a crystallization which regularly imbeds into $\mathbf{S}^{2}$, are proved to be homeomorphic to $\mathbf{S}^{n}$. A conjecture implying the Poincare Conjecture in dimension four is also formulated.
\end{abstract}

SunTo. Si dimostra che tutte le $n$-varietà di genere regolare zero, ciò̀ aventi una cristallizzazione che si immerge regolarmente in $\mathbf{S}^{2}$, sono omeomorfe a $\mathbf{S}^{n}$. Si formula anche una congettura che implica quella di Poincaré in dimensione quattro.

1. Throughout this paper, we work in the PL category, for which we refer to [RS]; for graph theory, we refer to [Har]. $\cong$ denotes PL-homeomorphism.

An $h$-coloured graph $(\Gamma, \gamma)$ is a multigraph $\Gamma$, regular of degree $h$, together with a coloration $\gamma$ of the edges by $h$ colours. If $\mathcal{T C}$ is the colour set, and $\mathscr{B} \subset \mathcal{K}, \Gamma_{\mathscr{B}}$ will denote the subgraph of $\Gamma$ generated by the edges e such that $\gamma(e) \in \mathscr{B}$. Given a colour $c \in \mathcal{K}, \hat{c}$ will denote the set $\mathcal{H}-\{c\}$. An $h$-coloured graph $(\Gamma, \gamma)$ is said to be contracted if $\Gamma_{\hat{c}}$ is connected for each $c \in \mathcal{F C}$.

To every $(n+1)$-coloured graph $(\Gamma, \gamma)$, there corresponds an $n$-dimensional pseudocomplex $K(\Gamma)$, whose $i$-simplexes are in one-one correspondence with the connected components of the subgraphs $\Gamma_{\mathscr{B}}$ for all colour subsets $\mathscr{B}$ of cardinality $\# B=n-i$. Note that, if $(\Gamma, \gamma)$ is contracted, then $K(\Gamma)$ has exactly $n+1$ vertices. For every closed, connected $n$-manifold $M$, there exists at least one contracted $(n+1)$-coloured graph $(\Gamma, \gamma)$ such that $|K(\Gamma)| \cong M$; such a graph is called a crystallization of $M$, and $K(\Gamma)$ a contracted triangulation of $M$. For the existence and equivalence theorems for crystallizations, see $\left[\mathbf{P}, \mathbf{F}, \mathbf{F G}_{\mathbf{1}}\right]$; these and other results are also summarized in [FGG].

We recall the notion of regular genus of a manifold, defined in $\left[\mathbf{G}_{3}\right]$, which generalizes the genus of a surface and Heegaard genus of a 3-manifold. A 2-cell imbedding [Wh, p. 40] $\iota:|\Gamma| \rightarrow F$ of an $(n+1)$-coloured graph $(\Gamma, \gamma)$ into a closed surface $F$ is said to be regular if there exists a cyclic permutation $\varepsilon=\left(\varepsilon_{0}, \ldots, \varepsilon_{n}\right)$ of the colour set, such that each region of $\iota$ is bounded by the image of a cycle, whose edges are alternatively coloured by $\varepsilon_{i}, \varepsilon_{i+1}(i$ being an integer $\bmod n+1)$. The regular genus $\rho(\Gamma)$ of $(\Gamma, \gamma)$ is defined to be the least genus of a surface into which

Received by the editors April 23, 1981.

1980 Mathematics Subject Classification. Primary 57N15, 57Q99; Secondary 05C10, 05C15, 57Q15.

Key words and phrases. PL-manifold, genus, Heegaard genus, multigraph, 2-cell imbedding, regular genus, crystallization, pseudocomplex, generalized Poincaré Conjecture.

1 The work was performed under the auspices of the G.N.S.A.G.A. of the C.N.R. (National Research Council) of Italy. 
$(\Gamma, \gamma)$ regularly imbeds. Given a closed $n$-manifold $M$, its regular genus (or simply genus) $\mathcal{G}(M)$ is defined as the integer

$$
\mathcal{G}(M)=\min \{\rho(\Gamma) \mid(\Gamma, \gamma) \text { is a crystallization of } M\} .
$$

As usual, we shall identify a graph with its imbedded image.

[ $\mathbf{G}_{3}$, Corollary 7] asserts, among other things, that a 4-manifold of genus zero is simply-connected. We shall extend this result to dimension $n$. This permits us to compute $\mathcal{S}\left(\mathbf{S}^{1} \times \mathbf{S}^{n}\right)$, and further to prove the following fact, which confirms the geometrical significance of this invariant.

THEOREM 1. Let $M$ be a closed, connected n-manifold; then

$$
\mathcal{G}(M)=0 \Leftrightarrow M \cong \mathbf{S}^{n} .
$$

REMARK 1. In view of Theorem 1, it would be interesting to study the behaviour of $\mathcal{G}$ with respect to connected sums. $\mathcal{G}$ is easily proved to be subadditive by direct construction. It is trivially additive in dimension 2 ; in dimension 3, the Heegaard genus-hence also the regular genus-is known to be additive too [Hak, §7]. If the same property held in dimension 4 , as we conjecture, this would imply an affirmative answer to the 4-dimensional Poincare Conjecture. In fact, as it is well known [M, \$1.1; Wa; C], if $M$ is a 4-dimensional homotopy sphere then, for a suitable nonnegative integer $k, M \sharp k\left(\mathbf{S}^{2} \times \mathbf{S}^{2}\right) \cong \mathbf{S}^{4} \sharp k\left(\mathbf{S}^{2} \times \mathbf{S}^{2}\right)$. But this would imply that $\mathcal{S}(M)=0$, whence $M \cong \mathrm{S}^{4}$.

We wish to thank M. Pezzana for the helpful discussions and ideas.

2. From now on, $\Delta_{n}=\{i \in \mathbf{Z} \mid 0 \leqslant i \leqslant n\}$ will be assumed as a colour set. For each $\mathscr{B} \subset \Delta_{n}, g_{\mathscr{B}}$ will denote the number of connected components of $\Gamma_{\mathscr{B}}$.

LEMMA 1. Let $(\Gamma, \gamma)$ be a contracted $(n+1)$-coloured graph, such that $\rho(\Gamma)=0$, and $\varepsilon=\left(\varepsilon_{0}, \ldots, \varepsilon_{n}\right)$, a cyclic permutation of $\Delta_{n}$ associated to a regular imbedding 1 of $(\Gamma, \gamma)$ into $S^{2}$. Let $\mathscr{B} \subset \Delta_{n}$ contain at least three colours $\varepsilon_{i-1}, \varepsilon_{i}, \varepsilon_{i+1}$ consecutive in $\varepsilon$ (i taken in $\mathbf{Z}_{n+1}$ ). Then $\mathrm{g}_{\mathscr{B}}=g_{\mathscr{B}-\left\{\varepsilon_{i}\right\}}$.

Proof. As $(\Gamma, \gamma)$ is contracted, $\Gamma_{\hat{\varepsilon}_{i}}$ is connected. Call $\gamma^{\prime}$ and $\iota^{\prime}$ the restrictions of $\gamma$ and $\iota$ respectively to the latter graph; then $\left(\Gamma_{\hat{\varepsilon}}, \gamma^{\prime}\right)$ is an $n$-coloured graph, regularly imbedded by $\iota^{\prime}$ into $S^{2}$. Namely, $\iota^{\prime}$ is a 2-cell imbedding [Wh, Theorem 6.11], and colours $\varepsilon_{i-1}, \varepsilon_{i+1}$ are now contiguous in the corresponding permutation of $\Delta_{n}-\left\{\varepsilon_{i}\right\}$; hence, $\left(\varepsilon_{i-1}, \varepsilon_{i+1}\right)$-coloured cycles bound regions of $\iota^{\prime}$.

Therefore, each edge coloured by $\varepsilon_{i}$ joins two vertices of the same component of $\Gamma_{\left\{e_{i-1}, e_{i+1}\right\}}$, thus also of the same component of $\Gamma_{\mathscr{B}^{-}-\left\{\varepsilon_{\mathrm{i}}\right\}}$.

LEMMA 2. Lei $(n, \gamma)$ and $\varepsilon$ be as in Lemma 1. Let further $\mathfrak{B}^{\prime}=\Delta_{n}-\mathscr{B}^{\prime}$, where $\mathfrak{B}^{\prime}$ contains no two colours consecutive in $\varepsilon$. Then $\mathfrak{g}_{\mathscr{B}}=1$.

Proof. Follows from Lemma 1, by induction on $\# \mathfrak{B}^{\prime}$.

Proposition 1. For a closed, connected n-manifold $M, \mathcal{S}(M)=0 \Rightarrow M$ is simplyconnected. 
Proof. Obvious for $n=2$. For $n>2$, if $(\Gamma, \gamma)$ of Lemma 2 is a crystallization of $M$, and $\mathscr{B}=\Delta_{n}-\{i, j\}$ with $i$ and $j$ not consecutive in $\varepsilon$, then there is only one component of $\Gamma_{\mathscr{B}}$. Then $\left[\mathbf{G}_{\mathbf{2}}, \S 6\right.$, Proposition 9] proves the statement.

As conjectured in $\left[\mathbf{F G}_{2}, \S 6\right]$, we have

Corollary 1. $\mathcal{G}\left(\mathbf{S}^{1} \times \mathbf{S}^{n}\right)=1$.

Proof. $\mathcal{G}\left(\mathbf{S}^{1} \times \mathbf{S}^{n}\right)>0$ by Proposition 1 .

In order to see that $\mathcal{G}\left(\mathbf{S}^{1} \times \mathbf{S}^{n}\right) \leqslant 1$, consider the following construction of a crystallization of $\mathbf{S}^{1} \times \mathbf{S}^{n}$, which generalizes [ $\mathbf{G}_{2}$, Figures 1,8] [FG $\mathbf{F}_{2}$, Figures 4, 7] and is obtained by applying the method illustrated in [FG $\left.\mathbf{F}_{2}, \S 2\right]$.

Take $2 n+4$ vertices $v_{j}^{i}\left(i \in \Delta_{1}, j \in \Delta_{n+1}\right)$. Join $v_{j}^{i}$ with $v_{j+1}^{i}\left(i \in \Delta_{1}, j \in \Delta_{n+1}\right)$ by an edge coloured by $j$. Put a further edge coloured by $n+1$ between $v_{0}^{i}$ and $v_{n+1}^{i}$ ( $i \in \Delta_{1}$ ) if $n$ is even, between $v_{0}^{0}$ and $v_{n+1}^{1}$ and between $v_{0}^{1}$ and $v_{n+1}^{0}$ if $n$ is odd. Finally, join $v_{j}^{0}$ with $v_{j}^{l}\left(j \in \Delta_{n+1}\right)$ by $n$ edges coloured by the $n$ colours not yet used around those vertices.

The fact that such a graph can be regularly imbedded into the torus-with respect to every cyclic permutation of $\Delta_{n+1}$-follows from the equality $\mathfrak{g}_{\{i, j\}}=n$ for all $i, j \in \Delta_{n+1}, i \neq j$ (see [FGG, §5]).

3. Proof of Theorem 1. It is trivial to see that $M \cong \mathbf{S}^{n} \Rightarrow \mathcal{G}(M)=0$, as $\mathbf{S}^{n}$ admits a standard crystallization consisting of two vertices joined by $n+1$ differently coloured edges; this graph obviously imbeds regularly into $\mathbf{S}^{2}$ with respect to every cyclic permutation of $\Delta_{n}$.

The proof of the converse implication consists of some general considerations followed by three parts; relative to the cases (A) $n$ odd, (B) $n$ even and $\neq 4$, (C) $n=4$.

In the following construction, which was first introduced in $\left[\mathbf{G}_{1}\right], M$ is an arbitrary closed $n$-manifold (not necessarily of genus zero), $(\Gamma, \gamma)$ a given crystallization of it, and $K$ the relative contracted triangulation.

In the vertex set $V=\left\{v_{0}, \ldots, v_{n}\right\}$ of $K$, assume that $v_{i}$ corresponds to $\Gamma_{i}$. For each nonvoid subset $W$ of $V$, set $W^{\prime}=V-W$, and call $K_{W}$ the contracted subcomplex of $K$ generated by $W$. If $W=h+1$, then $\operatorname{dim} K_{W}=h$. Furthermore, if $\mathscr{B}$ is the subset of $\Delta_{n}$ such that $W=\left\{v_{i} \mid i \in \mathscr{B}\right\}$ and $\mathscr{B}^{\prime}=\Delta_{n}-\mathscr{B}$, then the number of $h$-simplexes of $K_{W}$ equals $g_{\mathscr{B}}$; this is easy to check. Now let $L$ be the largest subcomplex of $\operatorname{Sd} K$, disjoint from $\operatorname{Sd} K_{W} \cup \operatorname{Sd} K_{W} \cdot{ }^{2}$ Then $L$, whose space is a closed ( $n-1)$-manifold, splits $K$ into two complementary subcomplexes, $N_{W}$ and $N_{W^{\prime}}$ say, having $L$ as common boundary. Moreover, $\left|N_{W}\right|$ and $\left|N_{W^{\prime}}\right|$ are regular neighbourhoods, in $|K|$, of $\left|K_{W}\right|$ and $\left|K_{W^{\prime}}\right|$ respectively. Observe that, in dimension three, if $\sharp W=2$, then $\left(\left|N_{W}\right|,\left|N_{W^{\prime}}\right|\right)$ is a Heegaard splitting of $M$.

From now on, the hypothesis $\rho(\Gamma)=0$ will be assumed, and $\iota:|\Gamma| \rightarrow S^{2}$ will denote a regular imbedding of $(\Gamma, \gamma)$; w.l.o.g., $\iota$ can be assumed to be associated to the fundamental cyclic permutation $\varepsilon=(0,1, \ldots, n)$.

${ }^{2}$ Sd means "barycentric subdivision of"; it carries every pseudocomplex to a simplicial complex. 
(A) $n=2 r+1, r \geqslant 0$.

Set $\mathscr{B}=\{2 k+1 \mid 0 \leqslant k \leqslant r\}, \mathscr{B}^{\prime}=\Delta_{n}-\mathscr{B}$; call $W, W^{\prime}$ the corresponding subsets of $V$. By Lemma $2, \mathfrak{g}_{\mathscr{B}^{\prime}}=\mathfrak{g}_{\mathscr{B}}=1$, whence $K_{W}$ and $K_{W^{\prime}}$ consist of exactly one $r$-simplex each. Therefore $\left|N_{W}\right|$ and $\left|N_{W^{\prime}}\right|$ are closed $(2 r+1)$-balls; they cover $M$, and meet in their common boundary $|L|$. Thus $M \cong \mathbf{S}^{2 r+1}$.

(B) $n=2 r, r \neq 2$.

$\mathscr{B}, \mathscr{B}^{\prime}, W, W^{\prime}$ as in case (A). Here, Lemma 2 only assures that $g_{\mathscr{B}^{\prime}}=1$, hence that $\left|N_{W}\right|$ is a $2 r$-ball. The $2 r$-complex $N_{W}$, whose boundary $L$ has a $(2 r-1)$-sphere as space, has the homotopy type of the $(r-1)$-complex $K_{W^{\prime}}$. These facts, applied to the Mayer-Vietoris homology sequence of $K=K_{W} \cup K_{W^{\prime}}$ and $L=K_{W} \cap K_{W^{\prime}}$, together with Poincare duality, imply that $M \cong|K|$ is a homology sphere. Therefore, as a consequence of Proposition 1 and of the Hurewicz isomorphism theorem, $M$ is even a homotopy sphere. This, which holds for all $r$, implies that $M \cong \mathbf{S}^{2 r}$ when $r \neq 2$, by the generalized Poincaré Conjecture (Smale, Stallings and Zeeman).

(C) $n=4$.

$\mathscr{B}=\{1,3\}, \mathscr{B}^{\prime}=\{0,2,4\} ; W, W^{\prime}$ as before. Again, $\mathfrak{g}_{\mathscr{B}^{\prime}}=1$ implies that $\left|N_{W}\right|$ is a 4-ball.

In order to show that $\left|N_{W^{\prime}}\right|$ is a 4-ball too, let us examine $K_{W^{\prime}}$ in some detail. Since $\mathfrak{g}_{\{1,3,4\}}=\mathfrak{g}_{\{0,1,3\}}=1$ by Lemma $2, K_{\left\{v_{0}, v_{2}\right\}}$ and $K_{\left\{v_{2}, v_{4}\right\}}$ are formed by one 1-simplex each. Hence all triangles forming $K_{W^{\prime}}$ have two edges in common; then $K_{W^{\prime}}$ will be a cone over the 1-pseudocomplex $K_{\left\{v_{0}, v_{4}\right\}}$ if it consists of as many triangles as there are edges in $K_{\left\{v_{0}, v_{4}\right\}}$. But this is actually the case, as $\mathfrak{g}_{\{1,2,3\}}=\mathfrak{g}_{\{1,3\}}$ by Lemma 1. Therefore $\left|K_{W^{\prime}}\right|$ is collapsible, $\left|N_{W^{\prime}}\right|$ is a 4-ball (by Whitehead's theorem [RS, Corollary 3.27]), and $M \cong \mathbf{S}^{4}$.

For $n \geqslant 2$ we have

COROllary $2_{n}$. Let $(\Gamma, \gamma)$ be a contracted $(n+1)$-coloured graph such that $\rho\left(\Gamma_{i}\right)=0$ for each $i \in \Delta_{n}$. Then $|K(\Gamma)|$ is a manifold.

Proof. For each $i \in \Delta_{n}, \Gamma_{\hat{i}}$ is connected and of regular genus zero. If $n=2, \Gamma_{\hat{i}}$ is a cycle and hence represents $\mathbf{S}^{1}$. If $n \geqslant 3$, the fact that $\left|K\left(\Gamma_{i}\right)\right| \cong \mathbf{S}^{n-1}$ is assured by Corollary $3_{n-1}$. This proves that, for each vertex $v$ of $K(\Gamma),|l k(v, \operatorname{Sd} K(\Gamma))| \cong$ $\mathbf{S}^{n-1}$, and this suffices to prove the statement (compare [F, Proposition 16]).

COROLlaRY $3_{n}$. Let $(\Gamma, \gamma)$ be a connected $(n+1)$-coloured graph such that $\rho(\Gamma)=0$. Then $|K(\Gamma)| \cong \mathbf{S}^{n}$.

Proof. By eliminating a suitable number of dipoles of type 1 [FG 1 , §3] one obtains a contracted graph $\left(\Gamma^{\prime}, \gamma^{\prime}\right)$. Now let $\iota:|\Gamma| \rightarrow \mathbf{S}^{2}$ be a regular imbedding of $(\Gamma, \gamma)$ into $\mathbf{S}^{2}$ relative to the cyclic permutation $\varepsilon$. Then by [FG $\mathbf{F}_{2}$, Lemma 1] there exists also an imbedding $\iota^{\prime}:\left|\Gamma^{\prime}\right| \rightarrow \mathbf{S}^{2}$ relative to the same $\varepsilon$.

If $\left|K\left(\Gamma^{\prime}\right)\right|$ is a manifold, i.e. if $\left(\Gamma^{\prime}, \gamma^{\prime}\right)$ is a crystallization, then $\left|K\left(\Gamma^{\prime}\right)\right| \cong|K(\Gamma)|$. But $\left|K\left(\Gamma^{\prime}\right)\right|$ is actually a manifold by Corollary $2_{n}$, since $\iota^{\prime}$ induces a regular imbedding of each $\left(\Gamma_{\hat{i}},\left.\gamma\right|_{\Gamma_{\hat{i}}}\right)$ into $\mathbf{S}^{2}$. Therefore $|K(\Gamma)| \cong\left|K\left(\Gamma^{\prime}\right)\right|=\mathbf{S}^{n}$ by Theorem 1 applied to $\left(\Gamma^{\prime}, \gamma^{\prime}\right)$. 


\section{REFERENCES}

[C] S. S. Cairns, The manifold smoothing problem, Bull. Amer. Math. Soc. 67 (1961), 237-238.

[F] M. Ferri, Una rappresentazione della n-varietà topologiche triangolabili mediante grafi $(n+1)$-colorati, Boll. Un. Mat. Ital. B 13 (1976), 250-260.

[FG ${ }_{1}$ ] M. Ferri and C. Gagliardi, Crystallisation moves, Pacific J. Math. 98 (1982).

$\left[\mathrm{FG}_{2}\right] \ldots$, On the genus of 4-dimensional products of manifolds (to appear).

[FGG] M. Ferri, C. Gagliardi and L. Grasselli, A graph-theoretical representation of PL-manifolds-A survey on crystallizations (to appear).

[G, $\mathbf{G}_{1}$ C. Gagliardi, Spezzamenti alla Heegaard per varietà n-dimensionali, Boll. Un. Mat. Ital. A 13 (1976), 302-311.

$\left[\mathbf{G}_{2}\right]$, How to deduce the fundamental group of a closed n-manifold from a contracted triangulation, J. Combinatorics Information Syst. Sci. 4 (1979), 237-252.

$\left[\mathbf{G}_{3}\right] \ldots$ Extending the concept of genus to dimension n, Proc. Amer. Math. Soc. 81 (1981), 473-481.

[Hak] W. Haken, Some results on surfaces in 3-manifolds, Studies in Modern Topology, No. 5, Math. Assoc. Amer., Prentice-Hall, Englewood Cliffs, N.J., 1968, pp. 39-98.

[Har] F. Harary, Graph theory, Addison-Wesley, Reading, Mass., 1969.

[M] R. Mandelbaum, Four-dimensional topology: An introduction, Bull. Amer. Math. Soc. 2 (1980), $1-157$.

[P] M. Pezzana, Sulla struttura topologica delle varietà compatte, Atti Sem. Mat. Fis. Univ. Modena 23 (1974), 269-277.

[RS] C. Rourke and B. Sanderson, Introduction to piecewise-linear topology, Springer-Verlag, Berlin and New York, 1972.

[Wa] C. T. C. Wall, On simply-connected 4-manifolds, J. London Math. Soc. 39 (1964), 141-149.

[Wh] A. T. White, Graphs, groups and surfaces, North-Holland, Amsterdam, 1973.

Istituto di Matematica, Facoltì di Ingegneria, V. Claudio, 21, I 80125 Napoli, Italia 